\title{
LA ANTROPOLOGÍA FÍSICA APLICADA A LAS INCINERACIONES HUMANAS. LA TUMBA ROMANA DE ORIPPO, SEVILLA
}

\author{
PHYSICAL ANTHROPOLOGY APPLIED TO HUMAN CREMATIONS. \\ THE ROMAN TOMB OF ORIPPO, SEVILLA
}

\author{
por \\ JOSE ALCAZAR GODOY \\ ANTONIO MANTERO TOCINO
}

RESUMEN En este artículo se desarrolla la metodología antropológica sobre dos incineraciones procedentes de una tumba romana de Orippo (Sevilla), excavada en 1988 y datada en el siglo I DC. La tumba fue construida para albergar los huesos quemados de una joven de edad comprendida entre 13 y 15 años, como se deduce de la fusión epifisaria de algunos huesos y del desarrollo de la dentición. La tumba fue reutilizada, probablemente algún tiempo después, para depositar dos urnas de cristal que contenían los huesos quemados de una mujer de edad comprendida entre 40 y 50 años, que padecía artrosis vertebral y coxofemoral, así como hiperóstosis interna del frontal.

\begin{abstract}
In this paper we present the anthropological study of two incinerations. They were found in a roman tomb excavated in 1988 in Orippo (Sevilla), and dated in the I century AC. The tomb was built to contain the burnt bones of a young woman betwen 13 and 15 years of age, as it is concluded of the epiphyseal union of some bones and of the dental development. The tomb was used again, probably sometime later, in orden to deposit two glass urns that contained the burnt bones of a woman, 40 o 50 years old, with a vertebral and coxofemoral arthropathy, and also internal hyperostosis of the frontal bone.
\end{abstract}

\section{INTRODUCCION}

El análisis antropológico de los huesos calcinados procedentes de incineraciones permite conocer los cambios de la edad en función del crecimiento oseo y de su maduración durante la vida del individuo, el sexo, las patologías óseas, la esperanza de vida, etc. Conjugando estos resultados con los procedentes de la investigación arqueológica puede configurarse el modo de vida y ciertas pautas de comportamiento de las poblaciones estudiadas. 
Este trabajo recoge el estudio antropológico de una compleja tumba de incineración romana del siglo I DC procedente de Orippo, Dos Hermanas, Sevilla, excavada en mayo de 1988.

La tumba, situada a $1,40 \mathrm{~m}$. de profundidad, consiste en una fosa de ladrillos de $1,80 \times 0,60$ $\mathrm{m}$. de lado y 0,67 de profundidad, cubierta por tres bipedalis. Sobre ella se había construido, también con ladrillos, un arco de medio punto de $0,52 \mathrm{~m}$. de luz, cegado con tégulas.

En el interior de la fosa -que llamaremos enterramiento inferior- apareció un conjunto de huesos calcinados con abundante y rico ajuar: dos morteros de piedra, dos veneras de bronce, unguentarios, objetos de vidrio, etc., así como dos agujas de hueso. Por otra parte, bajo el arco de medio punto, se recuperaron juntas dos urnas funerarias globulares de vidrio, con restos de las fundas de plomo que las recubrían. Una estaba fragmentada (urna 1), la otra conservaba intacto el cristal (urna 2). En las proximidades de estas dos urnas había depositados objetos funerarios: un cuenco, una paleta de tocador, cuatro piezas de hierro y un vaso.

La excavación arqueológica evidencia que la tumba de incineración fue construida para albergar los huesos quemados que se depositaron en el enterramiento inferior, y permite hipotizar la reutilización de la misma con el fin de alojar dos urnas con huesos quemados bajo el arco de medio punto, espacio que en principio no parece diseñado para albergar restos humanos.

\section{ENTERRAMIENTO INFERIOR}

\section{Neurocráneo}

- 4 fragmentos del frontal. Uno, con la cresta frontal desarrollada, está verticalizado, rasgo femenino; mide: $47,9 \times 22,6 \mathrm{~mm}$.

- Fragmento del reborde orbitario superior izquierdo que conserva la sutura frontomalar. El borde es agudo. Mide: $24,5 \times 17,5 \mathrm{~mm}$.

- Fragmento de frontal derecho con parte de la línea del temporal muy poco marcada. Mide: $37 \times 30,4 \mathrm{~mm}$.

- Fragmento de parietal correspondiente a la cara interna; conserva restos de diploe. La tabla externa ha estallado como consecuencia del calor. Se observan las impresiones de los surcos de las arterias meníngeas. Mide: $44 \times 40,7 \mathrm{~mm}$.

- Fragmento de temporal izquierdo con los surcos arteriales señalados. Mide: $43,2 \times 28,5 \mathrm{~mm}$.

- 2 fragmentos de parietal, uno del vertex y el otro próximo a la sutura lambdática. Miden: $45 \times 41,8$ mm.; 55,1x45,2 mm. Espesor aproximado: $5,5 \mathrm{~mm}$.

- 2 fragmentos con dientes de sutura abiertos, uno de la sutura coronal próximo al temporal de $47,1 \times 32,7 \mathrm{~mm}$, y otro de la sutura lambdática, de $46,1 \times 26,7 \mathrm{~mm}$.

- Fragmento con parte de la cavidad glenoidea que conserva la apófisis cigomática de $35,7 \mathrm{~mm}$. de longitud.

- Fragmento de occipital con la protuberancia occipital interna y parte de los canales del seno lateral y longitudinal superior. La cresta occipital externa está poco de-sarrollada y el inion es reducido, características femeninas (Cobb, 1952; Acsádi y Nemerskéri, 1970). Mide: 38,1 x 34 mm. Espesor en el inion $8,9 \mathrm{~mm}$.

- 11 fragmentos de calota craneal de pequeño tamaño y poco significado morfológico. 


\section{Esplacnocráneo}

- Fragmento de malar.

- Fragmento maxilar con parte de la abertura nasal. Mantiene los alveolos dentales de I2, C, Pml y Pm2; son de pequeño tamaño.

- Corona en formación del tercer molar inferior izquierdo. Está completamente calcinada por la acción del fuego, pero ha permitido conocer la edad juvenil temprana del individuo (Ubelaker, 78).

- Cóndilo mandibular pequeño.

- 2 raíces dentales calcinadas y fragmentadas con el canal radicular visible en una de ellas.

\section{Huesos largos}

- 4 fragmentos diafisarios del húmero. El mayor es derecho; mide: $96,3 \mathrm{~mm}$. de longitud, espesor: $2,7 \mathrm{~mm}$. Los restantes son de pequeño tamaño.

- 10 fragmentos diafisarios de fémur. Uno conserva la línea áspera poco marcada, propia de un reducido paquete muscular típica del sexo femenino. La longitud de los mayores: $60,5 \mathrm{~mm}$., 53,3 mm. y $45 \mathrm{~mm}$. El espesor máximo es de $5,6 \mathrm{~mm}$.

- 2 epífisis distales de ambos fémures sin soldar. La izquierda conserva la tróclea femoral y los dos cóndilos; la derecha, el cóndilo interno y la tróclea, y en ambas la escotadura itnercondílea. La ausencia de fusión epifisaria distal en los fémures ha permitido fijar el límite superior de la edad de muerte (Flecker, 1932/33; Stewart, 1954; Angel y otros, 1986).

- 2 fragmentos de diáfisis de tibia. El mayor, de la tibia izquierda, mide: $88 \mathrm{~mm}$. de longitud, y $2,7 \mathrm{~mm}$. cerca del foramen nutricio. Es grácil y poco robusto.

- 14 fragmentos diafisarios de cúbito, radio y peroné, gráciles y de escasos centímetros de longitud.

- 16 fragmentos de tejido esponjoso. Algunos corresponden a zonas epifisarias sin fusionar. Debido al deterioro es imposible su identificación.

- 4 fragmentos de diáfisis de huesos largos de pequeño tamaño no identificables.

\section{Vértebras}

- Masa central con la faceta articular superior e inferior del atlas.

- Fragmento de cuerpo vertebral cervical. Diámetro transverso $15 \mathrm{~mm}$, altura: $9,5 \mathrm{~mm}$.

- 2 fragmentos de cuerpos vertebrales dorsales. Diámetro transverso: 18,6 mm., altura: 15,7 mm.; diámetro transverso: $19,2 \mathrm{~mm}$., altura: $16,3 \mathrm{~mm}$.

- Segundo cuerpo vertebral lumbar. Diámetro transverso: $26,7 \mathrm{~mm}$., altura: $24,6 \mathrm{~mm}$.

- Tercer cuerpo vertebral lumbar. Diámetro transverso: $28,8 \mathrm{~mm}$., altura: $25,7 \mathrm{~mm}$.

- Cuarto cuerpo vertebral lumbar. Diámetro transverso: $28,9 \mathrm{~mm}$., altura: $23,3 \mathrm{~mm}$.

- Quinto cuerpo vertebral lumbar. Diámetro transverso: 33,5 mm., altura: 21,8 mm.

En los cuerpos vertebrales lumbares se observa un aplastamiento de sus plataformas articulares, más acentuado en la quinta vertebra lumbar y algo menos en las restantes. Por esta razón las alturas de los cuerpos disminuye progresivamente. Es probable la existencia de escoliosis en la columna vertebral.

Aunque las caras articulares no tienen estriaciones en los bordes, del tamaño y aspecto se infiere una edad juvenil.

El color oscila entre negro grisáceo y gris. 


\section{Costillas}

- Varios fragmentos de costillas, la mayor parte abiertos por la acción del fuego, en los que no es posible medir la anchura ni la altura.

\section{Coxales}

- Hueso coxal derecho integrado por parte del cuerpo del ilion y del isquion con la cavidad cotiloidea y parte de la tuberosidad isquiática. La escotadura ciática mayor es abierta, claramente femenina. En la cavidad cotiloidea han fusionado el ilion y el isquion, indicador de la edad de esta joven, no inferior a los 13 años (Mckern y Stewart, 1957).

\section{Manos y pies}

- Fragmento de primera falange.

- 2 fragmentos distales pertenecientes, probablemente, a los segundos metacarpianos. En ninguno ha soldado la epífisis distal, con lo que aún se determina con más precisión el límite superior de la edad.

- 2 fragmentos distales de los primeros metatarsianos, izquierdo y derecho.

- Fragmento del astrágalo derecho.

\section{Clavículas}

- 3 fragmentos de reducido tamaño.

\section{Peso}

$\begin{array}{lr}\text { - Neurocráneo } & 148,69 \mathrm{gr} . \\ \text { - Esplacnocráneo } & 7,20 \mathrm{gr} . \\ \text { - Huesos largos } & 235,76 \mathrm{gr} . \\ \text { - Vértebras } & 53,08 \mathrm{gr} . \\ \text { - Costillas } & 22,36 \mathrm{gr} . \\ \text { - Coxales } & 51,21 \mathrm{gr} . \\ \text { - Manos y pies } & 13,93 \mathrm{gr} . \\ \text { - Clavículas } & 9,24 \mathrm{gr} . \\ \text { - Restos pequeños } & 240,09 \mathrm{gr} . \\ \text { - Peso total } & 781,56 \mathrm{gr} .\end{array}$

\section{Conclusiones}

La incineración corresponde a una mujer de edad comprendida entre 13 y 15 años. El sexo se ha determinado con certeza a partir de la abertura de la escotadura ciática mayor, el inion reducido, la verticalidad del frontal, la agudeza del reborde supraorbitario y de la gracilidad de los huesos largos. El límite inferior de la edad se ha deducido de la fusión de los cuerpos del ilion y del isquion en la cavidad cotiloidea, así como del desarrollo de la corona del tercer molar; el superior lo establecen la fusión epifisaria de los metacarpianos y de los extremos distales de los fémures. 
A pesar de la corta edad, la inclinación de las plataformas articulares de los cuerpos vertebrales como consecuencia del aplastamiento en uno de sus lados sugiere que padecían escoliosis vertebral.

Conviene señalar la abundancia de huesos quemados alojados en la fosa; esto podría sugerir que la joven fue incinerada en un lugar cercano a la tumba. Además la trituración poco cuidadosa permitió recuperar algunos fragmentos grandes.

De la riqueza del ajuar que acompañaba a los huesos y de la significativa construcción de la tumba de incineración se deduce que la joven difunta gozó en vida de un rango social elevado.

\section{URNA 1}

\section{Neurocráneo}

- Fragmento endocraneal que contiene parte del frontal y del parietal unidos por la sutura coronal, soldada en la tabla interna. Contiene restos de diploe. Mide 44,3×18,6 mm.

- Fragmento de frontal con cresta frontal; presenta hiperóstosis interna (Reich, 1986).

- Fragmento de frontal con la sutura frontocigomática y el inicio de la órbita ocular; es de fino contorno.

- 53 fragmentos de calota craneal en los que la acción del fuego ha producido la explosión del hueso, separando las tablas interna y externa. Poseen manchas rojizas y ocres. Los mayores miden: $36,7 \times 24,5 \mathrm{~mm}$.; $21,7 \times 26,8 \mathrm{~mm}$;; $31 \times 26,3 \mathrm{~mm}$. El espesor varía entre 2 y $6 \mathrm{~mm}$.

- 10 fragmentos con restos de sutura correspondientes, todos ellos, a la tabla externa. La mayoría contienen algo de diploe. Los mayores miden: 26,7x17,2 mm.; 29,9x16,4 mm. El espesor oscila entre 2 y $4 \mathrm{~mm}$.

- 4 fragmentos con sutura cerrada en el endocráneo y abierta en el exocráneo. Miden: 41x22 mm., espesor: 6,5 mm.; 35,9x25,7 mm., espesor: 7,3 mm.; 29,5x16,3 mm., espesor: $5 \mathrm{~mm}$.; 29x17 mm., espesor: $7,8 \mathrm{~mm}$.

Uno de los fragmentos conserva la coincidencia de la sutura lambdática con la sagital, abierta la primera y con inicio de sinóstosis la segunda.

- Fragmento de occipital y de temporal separados por la sutura mastooccipital. En la cara interna se observa parte del seno transverso, y en la externa el agujero mastoideo. Mide: 57,5x36,7 mm.; espesor: $11,8 \mathrm{~mm}$.

El grado de unión de las suturas craneales (Cobb, 1952; Acsádi y Nemeskeri, 1970), obliteradas endocranealmente y abiertas en su mayor parte a lo largo de la tabla externa, sugiere que se trata de un individuo adulto de edad no muy avanzada.

- Fragmento del exocráneo de parte del temporal con la apófisis mastoides. Mide 41x19,7 mm., espesor: 6,4 mm. En norma posterior se observa que tanto la ranura mastoidea como la apófisis mastoides están recogidas hacia el interior del cráneo, una característica que unida al tamaño de la apófisis evidencia sin duda que se trata de una mujer (Hoshi, 1962).

La coloración de los huesos del cráneo es predominantemente blanca, con presencia en algunos fragmentos del negro.

\section{Esplacnocráneo}

- Fragmento de hueso cigomático izquierdo de tamaño pequeño y morfología grácil.

- Fragmento del maxilar. 
- Fragmento mandibular con presencia de alveolos pertenecientes a cuatro incisivos y dos caninos.

- Fragmento mandibular derecho con los alveolos del C y Pm1.

- 2 raíces dentales de $\mathrm{C}$ y $\mathrm{Pm} 1$ de la hemimandíbula derecha.

\section{Huesos largos}

- 2 cabezas femorales incompletas en las que no es posible medir el diámetro. La mayor presenta en la unión con el cuello del fémur una intensa deposición ósea característica de una patología correspondiente a la artrosis coxofemoral (Reich, 1986). Son de color negro por combustión incompleta. Miden: 69,5x41,6 mm.; $39 \times 32,9 \mathrm{~mm}$.

- 15 fragmentos de diáfisis femorales de la cara anterior y posterior. Los mayores miden 53,9x21,5 mm., espesor: $5,9 \mathrm{~m}$.; $43 \times 22,4 \mathrm{~mm}$., espesor: $7,3 \mathrm{~mm}$. Cuatro fragmentos pertenecientes a la cara posterior presentan una línea áspera poco desarrollada propia del sexo femenino. Los mayores miden: 42x17,4 mm., espesor a nivel de la línea áspera: $7 \mathrm{~mm}$.; 27,9x14,9 mm., espesor a nivel de la línea áspera: $7,6 \mathrm{~mm}$. Predomina el color blanco con manchas grises.

- 10 fragmentos de diáfisis de tibia. El mayor corresponde a la tibia izquierda y contiene el foramen nutricio; en la línea del sóleo hay una excrescencia ósea. Los mayores miden: 79,4x22,6 mm., espesor: $6,2 \mathrm{~mm}$.; 62,9×22,9 mm., espesor: $4,5 \mathrm{~mm}$. El color varía entre negro y gris.

- 8 fragmentos de diáfisis de húmero. Los mayores miden: 78,3×23,8 mm., espesor: $2,9 \mathrm{~mm}$.; $83 \times 22,6 \mathrm{~mm}$., espesor: $3,8 \mathrm{~mm}$. Son de color blanco.

- 21 fragmentos de diáfisis de húmero, cúbito, radio y peroné. Son de tamaño reducido y de color gris y blanco.

- Fragmentos de tejido esponjoso epifisarios. Los mejor conservados pertenecen a las mesetas tibiales, a los cóndilos femorales y a la tróclea humeral.

\section{Vértebras}

- Fragmentos del atlas: masa lateral con faceta articular superior, arco anterior y tubérculo.

- Apófisis odontoides del axis.

- Fragmentos pequeños de carillas articulares, pedículo y de cuerpos vertebrales.

- 4 cuerpos vertebrales cervicales fragmentados. Miden: diámetro transverso: $25,1 \mathrm{~mm}$., altura: $16,2 \mathrm{~mm}$.; diámetro anteroposterior: $25,2 \mathrm{~mm}$., altura: 14,4 mm.; altura: 15,8 mm.; altura: 16,5 mm. En tres de ellos se observan osteofitos bien desarrollados sobre los bordes anteriores de las plataformas articulares del cuerpo. Además de corresponder estas formaciones a un individuo adulto (Stewart, 1958), indican una artrosis cervical.

\section{Costillas}

- 15 fragmentos pequeños de color blanco y gris azulados por combustión incompleta, abiertas en su mayoría por la acción del fuego.

\section{Manos y pies}

- Fragmento de calcáneo.

- Segunda falange de la mano.

- 2 fragmentos de la cabeza del metacarpo izquierdo y derecho.

El estado incompleto de los fragmentos impide la obtención de medidas. 


\section{Clavículas}

- Fragmento de $36 \times 11 \mathrm{~mm}$.

Peso

- Neurocráneo

- Esplacnocráneo

- Huesos largos

- Vértebras

- Costillas

- Manos y pies

- Clavículas

- Restos pequeños

- Peso total

$$
\begin{aligned}
135,09 & \text { gr. } \\
6,64 & \text { gr. } \\
267,38 & \text { gr. } \\
18,42 & \text { gr. } \\
12,98 & \text { gr. } \\
6,68 & \text { gr. } \\
4,62 & \text { gr. } \\
269,70 & \text { gr. } \\
721,51 & \text { gr. }
\end{aligned}
$$

\section{Conclusiones}

Restos quemados de un esqueleto femenino de edad comprendida entre 40 y 50 años. Se diagnostican dos patologías óseas: hiperóstosis interna del frontal y artrosis avanzada. La hiperóstosis es una enfermedad metabólica predominante en el sexo femenino; está asociada generalmente con cefaleas y desestabilizaciones hormonales de la mujer y aparece entre 40 y 60 años. La artrosis coxofemoral (Iscan y Loth, 1989) dificultaría una locomoción equilibrada; los osteofitos de las vértebras cervicales producirían dolores musculares.

Dentro de la urna se encontró una aguja de hueso y un unguentario de vidrio.

Esta incineración permitió estudiar la posibilidad de una deposición intencionada de los huesos. Con esta finalidad se dividió en seis capas de un centímetro cada una y se procedió al análisis de los fragmentos. Finalmente se comprobó la presencia de fragmentos pertenecientes a un mismo hueso en distintas capas de la incineración, por lo que se pudo concluir que una vez quemados y triturados, los huesos fueron introducidos al azar en el interior de la urna.

\section{URNA 2}

\section{Neurocráneo}

- 11 fragmentos muy pequeños del exocráneo, de $15 \mathrm{~mm}$. de tamaño aproximado de lado. Algunos mantienen abiertos los dientes de las suturas. Predomina la coloración gris y blanca.

\section{Esplacnocráneo}

- 3 raíces dentales: 12 inferior izquierdo, M3 superior izquierdo, fragmento de molar.

- Fragmento de cóndilo mandibular.

- Fragmento de malar. 
Peso

- Neurocráneo

- Esplacnocráneo

Restos pequeños
7,31 gr.

2,60 gr.

$235,38 \mathrm{gr}$.

\section{Conclusiones}

La interpretación sobre el contenido óseo de esta urna ofrecía inicialmente varios interrogantes: era una cantidad reducida y muy triturada, y había fragmentos de los que se podía inferir su pertenencia a un adulto. Tras un análisis posterior sobre cada fragmento, se comprobó que el conjunto de la incineración no representaba partes significativas de un esqueleto que hubieran sido recogidas, ni tampoco era el resultado de una recolección incompleta; se trataba de los residuos pequeños de otra incineración.

Finalmente se observó que varios fragmentos de la calota craneal podían parangonarse con los de la urna 1 , tanto por la abertura de los dientes de las suturas y de su dibujo como por la estructura de los huesos. La correlación entre ambas urnas se estableció definitivamente al unir, por el lugar de rotura, un fragmento de peroné de la urna 2 con otro de la urna 1; igual sucedió con dos fragmentos mandibulares; y la raíz del incisivo lateral inferior derecho recuperado en la urna 2 se ajustaba al alveolo mandibular de la urna 1. Por tanto, los huesos de esta incineración forman parte del mismo esqueleto depositado en la urna 1, que albergó los de mayor tamaño, mientras que la urna 2 acogió los residuales, el producto final de una selección.

\section{BIBLIOGRAFIA}

ACSADI, GY. y NEMESKERI, J. (1970): History of Human Life Span and Mortality. Akadémiai Kiadó. Budapest. ANGEL, J. L., SUCHEY, J. M., ISCAN, M. Y. y ZIMMERMAN, M. R. (1986): "Age at death from the skeleton and viscera", Dating and Age Determination in Biological Materials. Cromm Helm. London. 179220.

COBB, W. M. (1952): Cowdry's Problems af Ageing. Williams and Wilkins. Baltimore.

FLECKER, H. (1932/33): "Roentgenographic observations of the times of appearance of the epiphyses and their fusion with the diaphyses". J. Anat. 67: 118-164.

HOSHI, H. (1962): "Sex difference in the shape of the mastoid process in norma occipitalis and its importance to the sex determination of the human skull", Okajima's Folia Anat. Japonica 38: 309-317.

ISCAN, M. Y. y LOTH, S. R. (1989): "Osteological Manifestations of Age in the Adult", Reconstruction of Life From the Skeleton. Alan R. Liss. New York: 23-40.

MCKERN, T. W. y STEWART, T. D. (1957): "Skeletal age changes in young American males, analyzed from the standpoint of identification", Headqu. Q. M. Res. and Dev. Command Tech Rep EP-45.

REICHS, K. J. (1986): "Forensic Implications of Skeletal Pathology: Sex", Forensic Osteology. Charles C. Thomas. Springfield: $112-142$.

STEWART, T. D. (1954): "Evaluation of evidence from the skeleton", Legal Medicine. St. Louis: 407-450.

STEWART, T. D. (1958): "The rate of development of vertebral osteoarthritis in American Whites and its significance in skeletal age identification", The Leech 28: 144-151.

UBELAKER, D. H. (1978): Human Skeletal Remains. Aldine. Chicago. 